\title{
Aluminum Silicate Fibers Reinforced Wood-plastic Composites: A Strengthening Strategy Based on the Interfacial Interactions between Inorganic Fibers and Organic Agricultural Wastes
}

\author{
Wenfan Yu, ${ }^{a, b}$ Wenyu Lu, ${ }^{\text {a,b }}$ Xiangsheng Han, ${ }^{a, b, *}$ and Hongzhen Cai ${ }^{\text {a,b,* }}$ \\ Using the methods of high-speed mixing, screw extrusion, and injection \\ molding, wood-plastic composites (CS-PF-ASF $x$ ) with inorganic fibers \\ (aluminum silicate fibers, ASF) and organic agricultural wastes (cotton \\ stalk, CS) as reinforcing fillers were prepared. The effects of different \\ contents of ASF on the properties of wood-plastic composites were \\ analyzed. Materials were characterized by Fourier infrared spectroscopy \\ (FT-IR), X-ray diffraction (XRD), scanning electron microscopy (SEM), and \\ thermogravimetric (TG) analyses. The creep, relaxation, and mechanical \\ behavior were also tested. With the increase of ASF contents, the FT-IR \\ peaks at approximately $1740 \mathrm{~cm}^{-1}$ shifted to lower wavenumber, indicating \\ the potential interactions between ASF and CS. The XRD patterns of the \\ composites implied that the crystal structures of each component were \\ maintained. Moreover, the addition of ASF enhanced the mechanical \\ properties and thermal stabilities of CS-PF-ASF $x$ composites, and the \\ tensile strength and impact strength reached maximum of approximately \\ $39.4 \mathrm{MPa}$ and approximately $3.89 \mathrm{~kJ} / \mathrm{m}^{2}$, respectively.
}

Keywords: Agriculture wastes; Aluminum silicate fibers; Recyclability; Interfacial interactions; Mechanical properties

Contact information: a: School of Agricultural Engineering and Food Science, Shandong University of Technology, Zibo, 255000, China; b: Shandong Research Center of Engineering and Technology for Clean Energy, Zibo, 255000, China; *Corresponding authors: hanxs@sdut.edu.cn; chzh666666@126.com

\section{INTRODUCTION}

Wood-plastic composites constructed with agricultural residues (e.g., cotton stalk, corn stalks, and peanut shells) and thermoplastics (e.g., polyolefin, high-density polyethylene, and polypropylene) have been demonstrated as a feasible strategy to recycle agricultural wastes into functional materials (Hietala et al. 2011; Schwarzkopf et al. 2017). The obtained composites are advantageous in their high strength, low density, and promising stability, which are widely used in the areas of building, furniture, and automotive interior panels (Liu et al. 2019; Sun et al. 2019).

In recent years, the mechanical or thermodynamic properties of wood-plastic composites have gained long-term developments. In particular, the impact of inorganic fillers in reinforcing wood-plastic composites has been remarkable, and this has aroused the research interest of scholars. Biomass, inorganic fibers, and polyethylene interact to form a three-dimensional spatial structure. This three-dimensional framework is conducive to improving the strength of composites. Meanwhile, it may induce the generation of crazing and shear bands. 
The stress field at the tip of the crazing triggers shear bands, and the shear bands can counteract the crazing and weaken the development of cracks. Repeated occurrence of this process can absorb a lot of energy and increase the strength of the material (Bucknall 2001). In addition, inorganic fillers have many advantages such as strong heat resistance and good thermal stability, which play a role in resisting heat transfer in composites and improve the thermodynamic properties of the materials. For example, due to the high mechanical properties, heat resistance, and chemical resistance of basalt fibers, the basalt fibers reinforced wood-plastic composites exhibited higher flexure, tensile, and dynamic modulus than wood-plastic composites without basalt fibers (Czigány 2006; Wu et al. 2014). Glass fibers enhanced the tensile strength, flexural strength, and flame retardancy of the composites due to their insulation properties, heat resistance, corrosion resistance, and high mechanical strength (Guo and Kethineni 2020). Silica particles reinforced the mechanical properties and thermal stability of wood-plastic composites based on their unique advantages of strong hardness and high melting point (Hao et al. 2018). Typically, the addition of inorganic fillers can affect the interfacial combination of wood-plastic composites and endow the materials with specific functionalities that can be attributed to their surface properties (i.e., oxygen-containing group). Thus, the introducing of inorganic fillers into wood-plastic composites and the relevant enhancing mechanism are meaningful issues in materials science.

Aluminum silicate fiber (ASF) is a kind of cotton filamentous inorganic fiber, containing mainly kaolin, alumina, and silica (Yang et al. 2013). It not only has advantages of thermal stability, mechanical properties, strong corrosion resistance, but it also is cheap and easy to prepare.

As insulation and heat insulation materials, after weaving, aluminum silicate fiber rope, aluminum silicate fiber board, or aluminum silicate fiber blanket can be fabricated and widely used by various thermal equipment, such as electric power and machinery, and by the chemical industry for heat preservation (Zhao et al. 2012; Sun et al. 2014). With the wide usage of aluminum silicate fiber materials, large amounts of wasted aluminum silicate fibers are produced and need to be efficiently recycled.

This study proposes to use discarded one-dimensional aluminum silicate fibers as inorganic fillers to enhance the mechanical properties and thermal stability of wood-plastic composites. Herein, the aluminum silicate fibers-polyethylene-cotton stalk particles composites (CS-PF-ASFx) were prepared by high-speed mixing, screw extrusion, and injection molding.

The inorganic filler ASF has strong heat resistance, and its uniform dispersion in the material improves the thermal stability of the composites. The tensile strength and impact strength of the composites reached maximum of approximately $39.4 \mathrm{MPa}$ and approximately $3.89 \mathrm{~kJ} / \mathrm{m}^{2}$, respectively. The enhancing mechanism was ascribed to the uniform dispersion of aluminum silicate fibers in wood-plastic composites and the potential hydrogen bonding between cotton stalk and oxygen-containing groups on the surface of aluminum silicate fibers. The promising interfacial interactions thus optimized the interfacial binding among CS, ASF, and HDPE, which was dominated in the mechanical and thermal properties of wood-plastic composites. This work provides a friendly, simple, and low-cost strategy to converting construction and agriculture wastes into functional wood-plastic materials. 


\section{EXPERIMENTAL}

\section{Materials}

The thermoplastic matrix used in this study was high-density polyethylene, which was purchased from Taiwan Plastic Co., Ltd. (Taiwan, China), and its grade was 9001. The density of high-density polyethylene is $0.950 \mathrm{~g} / \mathrm{cm}^{3}$, and the melt flow index is $0.05 \mathrm{~g} / 10$ min. The aluminum silicate fibers were purchased from Langfang Zhongying Asbestos Chemical Co., Ltd. (Langfang, China), and milled as powders using a frozen crusher (DC31; Shijiazhuang Benchen Mechanical and Electrical Equipment Co., Ltd., Shijiazhuang, China). Cotton stalk, produced in Liaocheng, China, were ground into particles and passed through 80-mesh sieve. The element composition of ASF and CS is shown in Table 1. Compatibilizer (maleic anhydride-grafted-polyethylene, MAPE) was purchased from Anhui Guofeng Wood-Plastic Composites Co., Ltd. (Hefei, China). The PE WAX (DH106) was purchased from Dongguan Dinghai Plastic Chemical Co., Ltd. (Dongguan, China).

\section{Sample Preparation}

The preparation processes of CS-PE-ASFX composites are shown in Fig. 1. The HDPE, CS, and ASF were used to prepare composites. As biomass, the CS fibers and the polar groups $(-\mathrm{OH},-\mathrm{COOH}$, etc. $)$ on their surfaces have been shown to play important roles in the preparation of composites (Yang et al. 2019). The polar groups can hydrogen bond with oxygen containing groups on reinforcing agents and inhibit the aggregation of ASF by weakening their intrinsic noncovalent interactions (Wu et al. 2018). Such interactions facilitated the uniform distribution of fillers and improved the interfacial binding among components in wood-plastic composites.

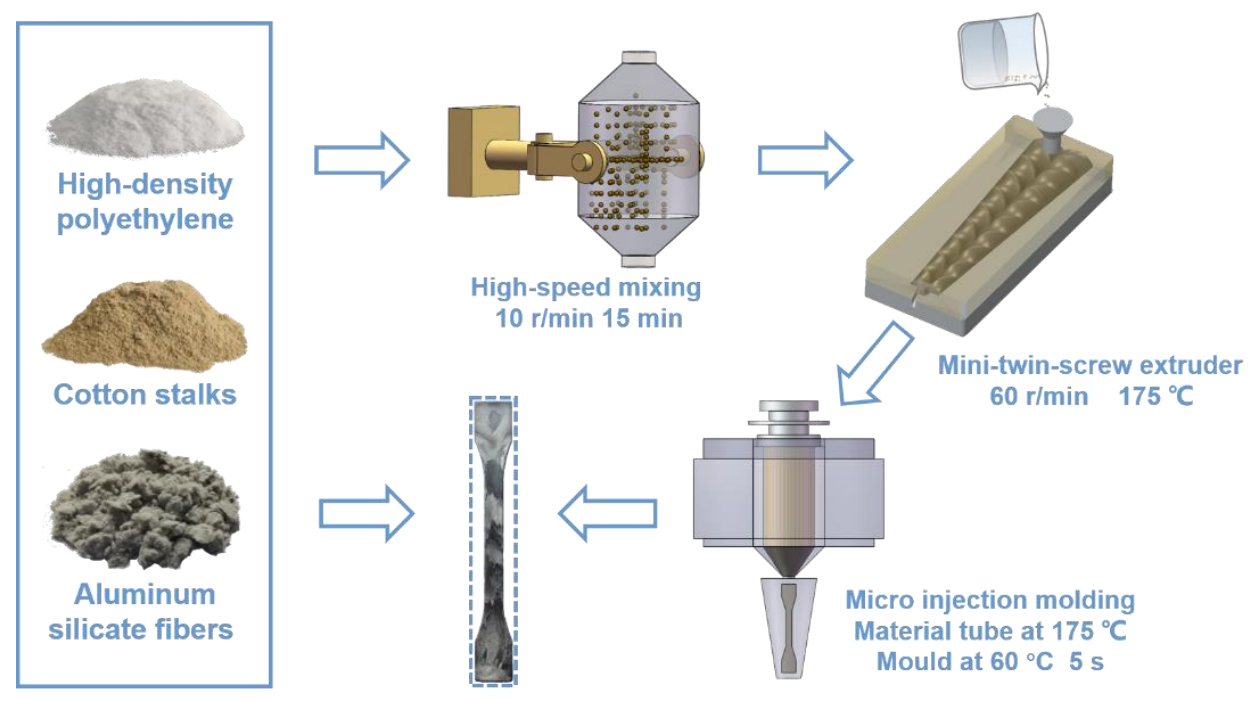

Fig. 1. Schematic illustration of fabrication of CS-PE-ASFx composites

Typically, the aluminum silicate fibers and cotton stalk were dried at $100{ }^{\circ} \mathrm{C}$ for 24 $\mathrm{h}$ to remove excess moisture. Thereafter, the ASF, CS, HDPE, MAPE, and PE WAX were mixed in a high-speed mixer at $10 \mathrm{r} / \mathrm{min}$ for $15 \mathrm{~min}$ to obtain uniform mixture. The uniform mixture was melted and mixed in a micro twin-screw extruder (WLG10G; Shanghai Xinshuo Precision Machinery Co., Ltd., Shanghai, China), and then the various test splines 
were molded using the micro injection molding machine (WZS10D; Shanghai Xinshuo Precision Machinery Co., Ltd., Shanghai, China). The upper and lower plate temperature and extrusion speed of the micro twin-screw extruder were $175{ }^{\circ} \mathrm{C}$ and $60 \mathrm{r} / \mathrm{min}$, respectively. The barrel temperature, material tube temperature, and holding time of the injection molding machine were set at $175{ }^{\circ} \mathrm{C}, 60{ }^{\circ} \mathrm{C}$, and $5 \mathrm{~s}$, respectively. The experimental formulation is shown in Table 1.

Table 1. Experimental Formulation Design (wt\%)

\begin{tabular}{|c|c|c|c|c|c|}
\hline Type & ASF & CS & HDPE & MAPE & PE WAX \\
\hline CS-PE-ASF $_{0}$ & 0 & 60 & 30 & 5 & 5 \\
\hline CS-PE-ASF $_{10}$ & 10 & 50 & 30 & 5 & 5 \\
\hline CS-PE-ASF $_{20}$ & 20 & 40 & 30 & 5 & 5 \\
\hline CS-PE-ASF $_{30}$ & 30 & 30 & 30 & 5 & 5 \\
\hline CS-PE-ASF $_{40}$ & 40 & 20 & 30 & 5 & 5 \\
\hline CS-PE-ASF $_{50}$ & 50 & 10 & 30 & 5 & 5 \\
\hline CS-PE-ASF $_{60}$ & 60 & 0 & 30 & 5 & 5 \\
\hline
\end{tabular}

\section{Characterization}

\section{FT-IR analysis}

The Fourier transform infrared (FT-IR) spectroscopy analysis of the composites was carried out using a Fourier transform infrared spectroscope (Nicolet 5700; Thermo Fisher Scientific, Waltham, MA, USA) via the KBr method. Spectra collected ranged from 4000 to $400 \mathrm{~cm}^{-1}$. Before FT-IR analysis, the composite samples were milled and washed with alcohol 3 times, and then dried at $60{ }^{\circ} \mathrm{C}$ for $24 \mathrm{~h}$.

\section{$X$-ray diffraction}

The crystal structure of the composites was evaluated using a Polycrystalline X-ray diffractometer (Bruker AXS D8 Advance, Karlsruhe, Germany). $\mathrm{Cu}-\mathrm{K} \alpha$ radiation was generated at $40 \mathrm{KV}$ and $50 \mathrm{~mA}$, with $2 \theta$ varying between $5^{\circ}$ and $60^{\circ}$ at $2 \% \mathrm{~min}$. Before the test, the samples were ground, then rinsed with alcohol 3 times, and dried at $60{ }^{\circ} \mathrm{C}$ for 24 h.

\section{Morphological observations of ASF and composites}

The microstructural analysis of the fracture surfaces of the ASF and composites were investigated using a field emission scanning election microscope (FE-SEM, FEI Sirion 200; Waltham, MA, USA). Before observing the sample, a layer of gold was sprayed (Vacuum Device Inc, Osaka, Japan) on the surface of the impact fracture. Energydispersive X-ray spectroscopy (FE-SEM, FEI Sirion 200; Waltham, MA, USA) mapping of broken samples were performed to verify the presence and distribution of ASF in composites.

\section{Mechanical properties}

Tensile specimens $\left(150 \times 10 \times 4 \mathrm{~mm}^{3}\right)$ were tested using an electronic universal testing machine (WDW1020; Changchun Kexin Co., Ltd., Changchun, China). The load cell of the machine was CELTRON STC-2T. The tensile test standard was GB/T 1040.12018. The stretching speed was $10 \mathrm{~mm} / \mathrm{min}$. The impact properties of the samples were tested using a pendulum electronic impact tester (JB-300b; Jinan Hengsi Shengda Instrument Co., Ltd., Jinan, China). The impact test standard was GB/T 1843-2008. The 
samples size was $80 \times 10 \times 4 \mathrm{~mm}^{3}$ and the impact energy was $1 \mathrm{~J}$. Five replicates of each formulation were tested.

\section{Creep and Relaxation Behavior}

A dynamic mechanical analyzer (Q800; TA Instruments, Baker, FL, USA) was used to test the creep and relaxation behavior of the samples $\left(35 \times 10 \times 4 \mathrm{~mm}^{3}\right)$. The timetemperature superposition (TTS) model was selected for the experiment, which was carried out at $35^{\circ} \mathrm{C}$, frequency of $1 \mathrm{~Hz}$, and strain of $0.1 \%$ for $30 \mathrm{~min}$. The pressure was kept at 1 $\mathrm{MPa}$, and the creep behavior of the samples was tested at $25{ }^{\circ} \mathrm{C}, 35^{\circ} \mathrm{C}, 45^{\circ} \mathrm{C}, 55^{\circ} \mathrm{C}$, and $65^{\circ} \mathrm{C}$ for $30 \mathrm{~min}$. Stress relaxation was tested by the same conditions to withstand a strain of $0.05 \%$.

\section{Thermal properties}

Thermal stability was evaluated with a synchronous thermal analyzer (STA 449; NETZSCH Scientific Instruments Trading Ltd., Selb, Germany) under the protection of a nitrogen atmosphere. The experiment was heated from 30 to $1100{ }^{\circ} \mathrm{C}$ at a heating rate of $10^{\circ} \mathrm{C} / \mathrm{min}$. The flow rate of nitrogen was $20 \mathrm{~mL} / \mathrm{min}$.

\section{RESULTS AND DISCUSSION}

\section{FT-IR Analysis}

Figure 2 shows the FT-IR spectra of CS, HDPE, ASF, and CS-PE-ASFx with different contents of ASF. The spectra were mainly used to evaluate the potential interactions among each of the components. The broad absorption peak of CS at 3100 to $3600 \mathrm{~cm}^{-1}$ was attributed to the stretching vibration of the $\mathrm{O}-\mathrm{H}$ and hydrogen bond in hydroxyl groups (Huang et al. 2020). The $\mathrm{C}-\mathrm{H}$ in $\mathrm{CH}$ and $\mathrm{CH}_{2}$ showed absorption peaks at 2923 and $2850 \mathrm{~cm}^{-1}$. The absorption peaks at 1741 and $1633 \mathrm{~cm}^{-1}$ were related to the vibration of $\mathrm{C}=\mathrm{O}$ and $\mathrm{C}=\mathrm{C}$ of the aromatic ring. Two small bands around 1510 and 1453 $\mathrm{cm}^{-1}$ were related to the stretching vibration of the aromatic skeleton in lignin, and the peak at $1376 \mathrm{~cm}^{-1}$ corresponded to the $\mathrm{C}-\mathrm{H}$ stretching vibration of cellulose and hemicellulose (Hsiao et al. 2010).
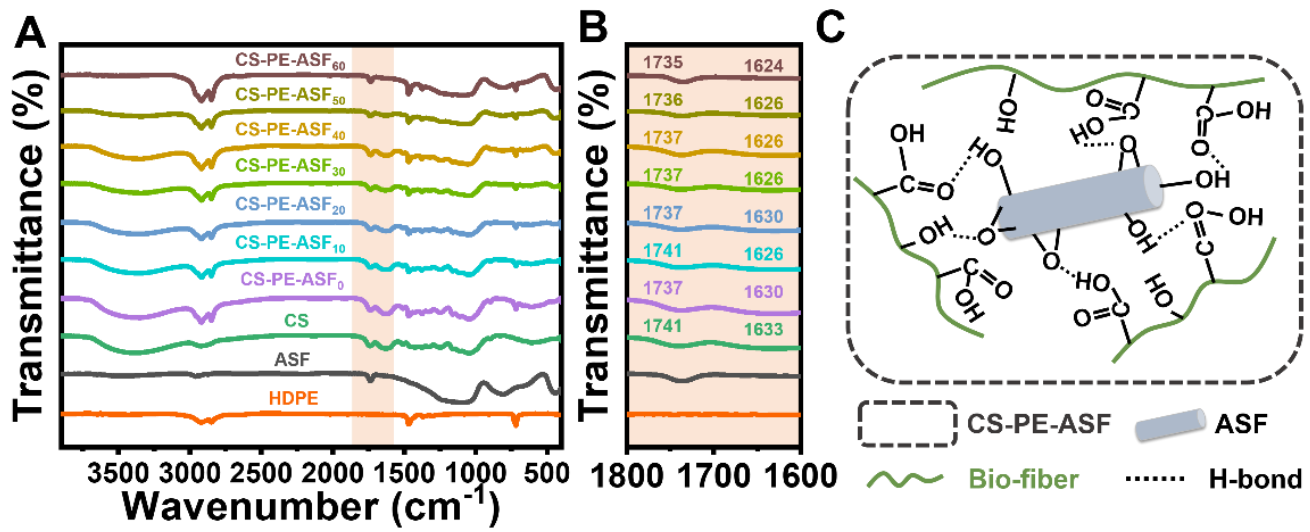

Fig. 2. (A) FT-IR spectra of CS, ASF, HDPE, and CS-PE-ASFx composites with different ASF contents; (B) FT-IR spectra of wavenumber form $1600 \mathrm{~cm}^{-1}$ to $1800 \mathrm{~cm}^{-1}$ emphasized the shift of characteristic peaks; (C) The possible hydrogen bonding between CS and ASF 
Figure 2B shows that with the gradual increase of ASF contents in the composites; the characteristic peaks of CS at $1741 \mathrm{~cm}^{-1}$ and $1633 \mathrm{~cm}^{-1}$ shifted into 1735 and $1624 \mathrm{~cm}^{-1}$. This indicated the changes of their chemical environments caused by the potential interactions among the components in CS-PE-ASFX composites. As shown in Fig. 2C, the oxygen-containing groups in aluminum silicate fibers can form hydrogen bonds with the hydroxyl hydrogen in CS, resulting in the shift of the characteristic peaks. The noncovalent interactions (hydrogen bond, $\pi-\pi$ stacking, and electrostatic interactions) on the interfaces in wood-plastic composites were crucial to the enhancement of materials properties, as they can regulate the interfacial binding situations and endow materials capabilities to resist external forces and invasions (Peri 1966; Armistead and Hockey 1967; Paul and Yates 1991; Slavov et al. 1996; Li and Matuana 2003; Zhang et al. 2020).

\section{X-ray Diffraction}

Figure 3 exhibits the XRD spectra of CS, HDPE, ASF, CS-PE-ASF 30 , and CS-PE$\mathrm{ASF}_{60}$. The diffraction peaks of HDPE at $21.6^{\circ}$ and $24^{\circ}$ corresponded to the (110) and (200) crystal planes, respectively. The CS peaks at $2 \theta=16^{\circ}$ and $22^{\circ}$ correspond to the amorphous structure and cellulose I crystals, respectively (Oudiani et al. 2011). No obvious diffraction peaks were found in aluminum silicate fibers, indicating the low crystallinity of waste ASF. It can be seen from the XRD spectra of CS-PE-ASF 30 that the diffraction peaks of HDPE and CS were maintained, indicating that the crystal structure of each component was not changed during the preparation process. However, compared with pure HDPE, the peak intensity of HDPE in CS-PE-ASF 30 was remarkably weakened. This was mainly attributed to the addition of fillers, which leads to a decrease in crystalline HDPE in composites (Zhang et al. 2019b).

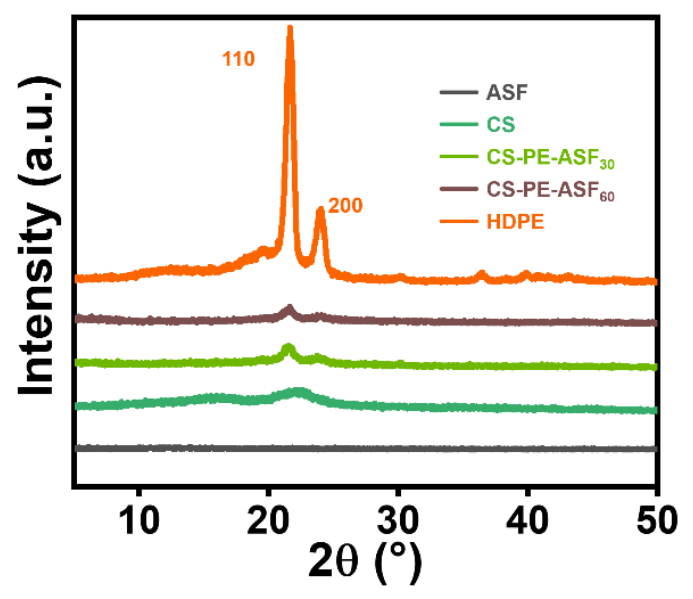

Fig. 3. XRD curves of ASF, CS, HDPE, CS-PE-ASF 30 , and CS-PE-ASF 60

\section{Morphological Observations of ASF and CS-PE-ASFx Composites}

Figures $4 \mathrm{~A}$ through $4 \mathrm{G}$ show the scanning electron microscope images of CS-PEASFx under different formulations. It can be seen from Fig. 4A that most of the composites were biomass particles, and HDPE served as a matrix for bonding between the interfaces and showed good interface bonding effects (Ou et al. 2014). When the contents of ASF was $10 \mathrm{wt} \%$, obvious cracks appeared at the cross section of the composites, indicating poor interfacial binding between fillers and polymer matrix (Fig. 4B). With the increase of ASF content, cracks and holes appeared in the composites, indicating that the combination 
of materials was further weakened. But when the content of ASF reached $50 \mathrm{wt} \%$, the combination of the components was obviously improved, and the composites became denser. As shown in Fig. 5, ASF were fibers with diameters between 500 and $5000 \mathrm{~nm}$, and their surfaces were smooth and flat. After injection molding, the surfaces of ASF were covered with a layer of polyethylene matrix, indicating tight and dense binding with polyethylene matrix. This promising adhesion thus facilitated the uniform and dense dispersion of ASF and promoted their materials properties (Zhang et al. 2019a).

Figure $4 \mathrm{H}$ and Fig. 4I show the energy dispersive X-ray spectrum (EDS) and mappings of CS-PE-ASF60, respectively. It should be noted that the distribution of silicon, oxygen, and aluminum in CS-PE-ASF 60 were homogenous, implying the presence and good dispersion of ASF in CS-PE-ASFX composites.

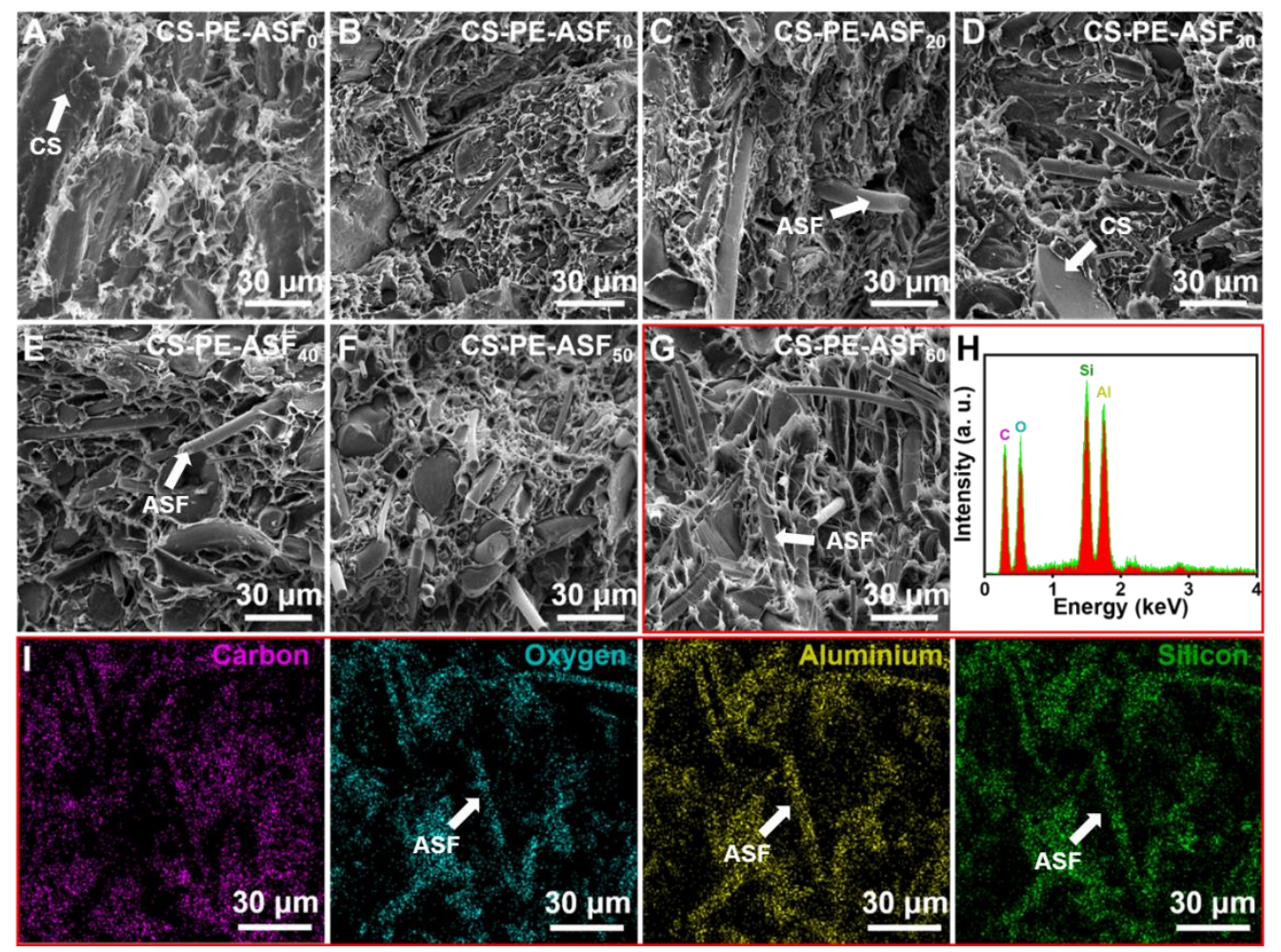

Fig. 4. Scanning electron micrograph of CS-PE-ASFx composites (A through $G$ ); EDS $(H)$ and mapping images of carbon, oxygen, silicon, and aluminum of CS-PE-ASF 60 composites (I)

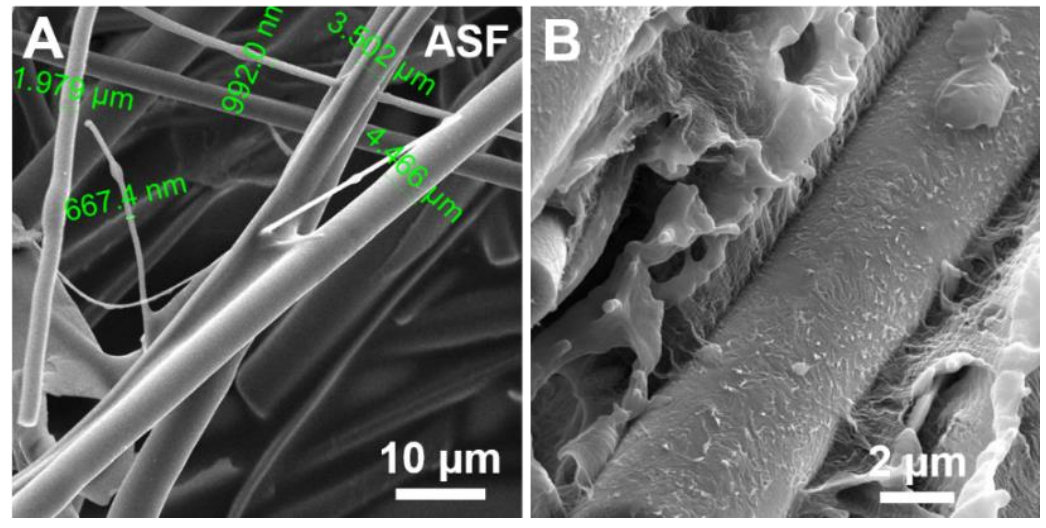

Fig. 5. Scanning electron micrograph of $A S F(A)$ and interfacial bonding of $A S F(B)$ 


\section{Mechanical Properties}

Figure 6 shows the tensile strength and impact strength of the CS-PE-ASFx composites. With the gradual increase of ASF contents in CS-PE-ASFx composites, the tensile strength and impact strength improved and reached a maximum of approximately $39.4 \mathrm{MPa}$ and approximately $3.89 \mathrm{~kJ} / \mathrm{m}^{2}$, respectively (Fig. 6A and 6B). Figure 6A illustrates that the changes of tensile strength were divided into two stages. The tensile strength gradually decreased with the additions of ASF from $10 \mathrm{wt} \%$ to $30 \mathrm{wt} \%$ stages. This may be attributed to the low content of ASF. It causes ASF to be sparsely dispersed inside the composites, which affects the interaction of the entire material and weakens the mechanical strength. However, the tensile strength was improved at higher ASF contents (30 wt\% to $60 \mathrm{wt} \%$ ), ascribed to the strengthened interfacial adhesion between ASF and polymer matrix and the hydrogen bonding between ASF and CS fibers. Especially when the content of ASF reaches $50 \%$, its internal distribution becomes denser, which can better transmit stress. Thus, the mechanical properties of the composites were significantly improved. When loaded by an external force, the tip part of the ASF will produce stress concentration and form micro-cracks. Microcracks induce the generation of shear bands, and the shear bands inhibit the development of microcracks. This repeated process consumes energy and improves impact strength. The greater impact strength implied the stronger toughness of the materials (Mittal et al. 2016). With more ASF addition, the impact strength of the composites was gradually promoted, demonstrating the improvement of the toughness of CS-PE-ASFx composites (Fig. 6B). As a onedimensional fiber, ASF has higher mechanical toughness and strong interface interaction with the polymer matrix, thus enhancing the toughness of CS-PE-ASFx composites.
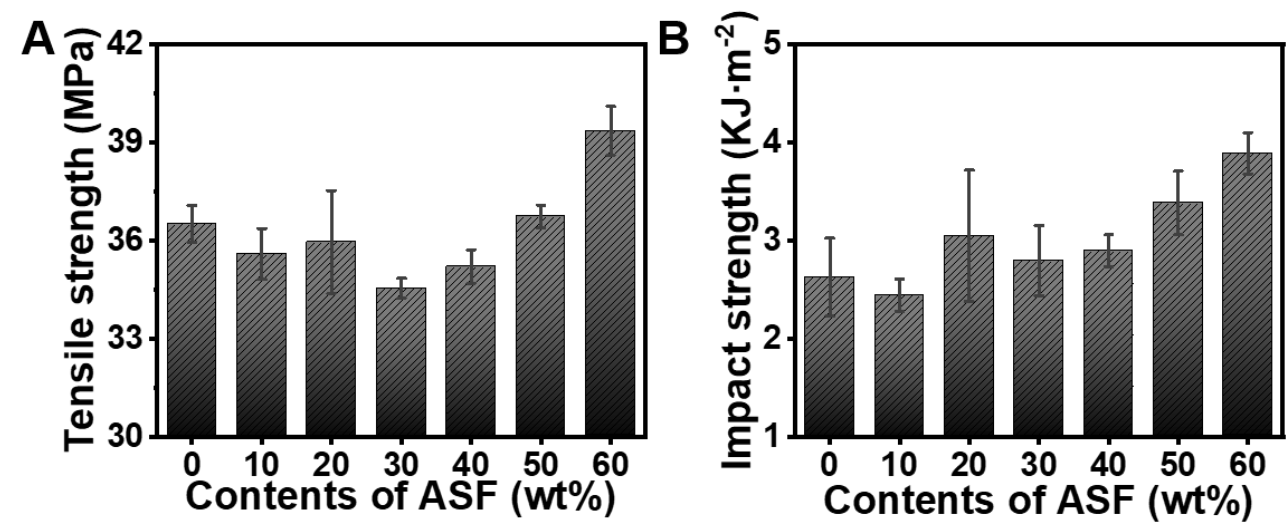

Fig. 6. The tensile strength (A) and impact strength (B) of CS-PE-ASFx composites

\section{Creep and Relaxation Behavior}

Figure 7 shows the creep and relaxation behavior of CS-PE-ASFx composites with different contents of ASF and different temperatures. The creep behavior of CS-PE-ASFX composites can be divided into three stages: the first stage was elastic deformation, the second stage was viscoelastic deformation, and the third stage was viscous deformation (Costa et al. 2016). It can be seen from Fig. 7A, compared with pure HDPE, the addition of filler greatly enhanced the creep resistance of the composites. Similarly, different temperatures will also affect the creep behavior of composites. As the temperature increased, the thermal kinetic energy and free volume of the matrix were improved, resulting in an increase in the creep compliance of the composites and a decrease in the 
creep performance (Zhang et al. 2017). On the side, the addition of fillers also increased the stress relaxation modulus of the composites (Fig. 7C). The strengthening of the stress relaxation modulus indicated the improvement of anti-stress relaxation ability of CS-PEASFx composites. The relaxation modulus of composites at different temperatures obeyed the general law. As the temperature increased, the degree of freedom between molecules increased, the stress declined correspondingly, and the relaxation modulus also decreased (Wang et al. 2017).
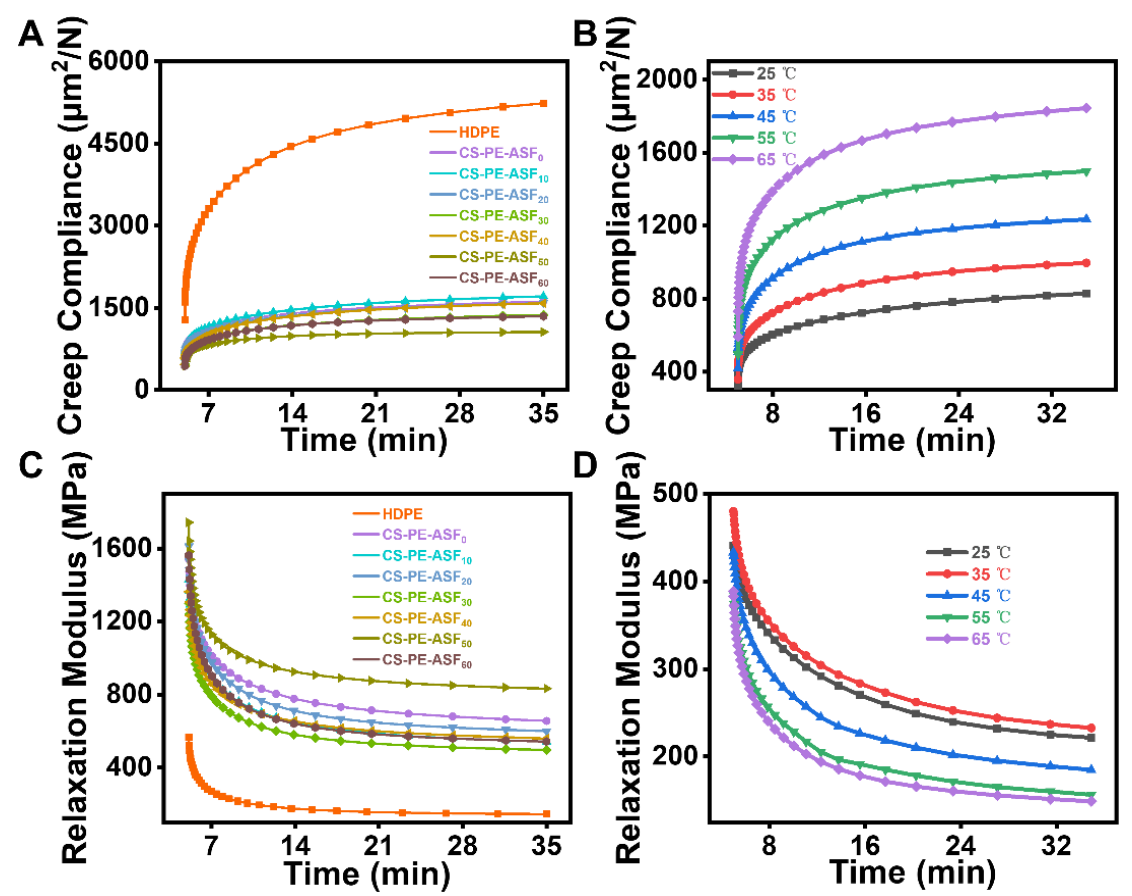

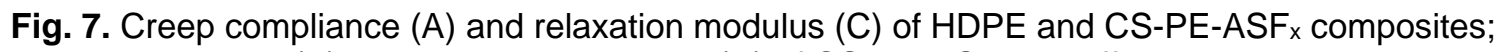
creep compliance $(B)$ and relaxation modulus $(D)$ of $C S-P E-A S F 30$ at different temperatures

\section{Thermal Properties}

To study the thermal properties of the CS-PE-ASFx composites, weight loss and weight loss derivatives were studied (Fig. 8). It can be seen from the weight loss curves in Fig. 8A that the CS-PE-ASFx composites had two thermal decomposition stages. In the first stage, the hemicellulose and $\alpha$-cellulose in the composites began to depolymerize, and the glycosidic bonds in the cellulose began to break. In the second stage, the carbon-carbon bonds of the polymers in the composites began to break, with the formation of volatile compounds, and the lignin in the CS also began to decompose (Moreno and Saron 2017). When the ASF contents gradually increased, the weight loss of the composites decreased, which was closely related to the better thermal stability of ASF. Its degradation temperature was higher than cotton fiber. The temperature above $200{ }^{\circ} \mathrm{C}$ was appropriate for burning of cotton fiber. As a result of this, weight loss was higher when the cotton fiber content was high. The hydrogen bonding between ASF and CS, and the uniform dispersion of ASF also effectively prevent the reduction of composites mass loss. The thermal stable ASF in CS-PE-ASFX composites can inhibit the heat transformation and the penetration of volatile degradation products in the composites and improve the thermal stability of the CS-PEASFx composites (Koohestani et al. 2017). 

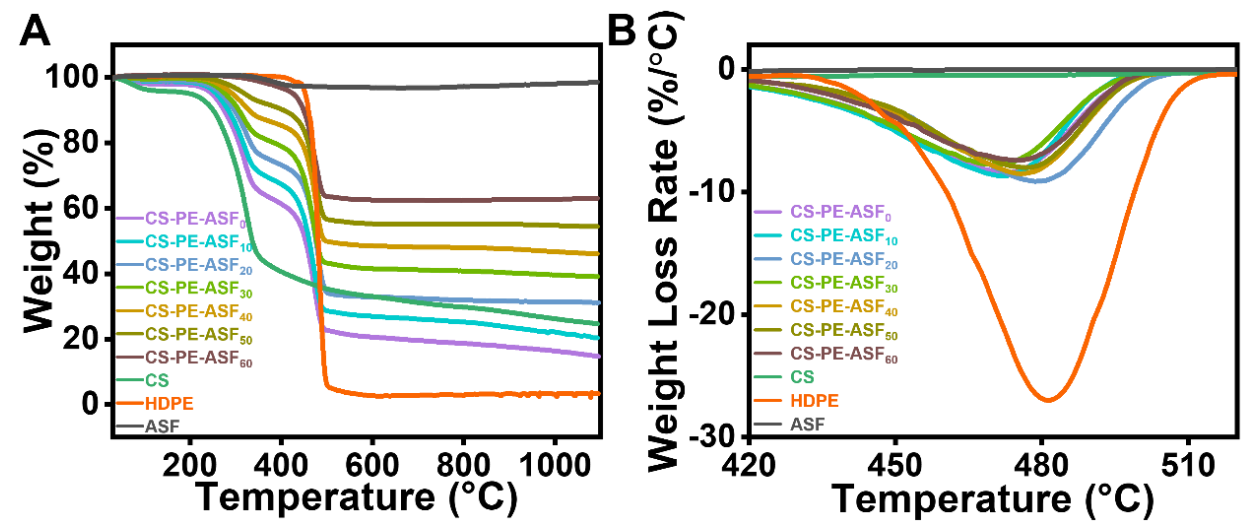

Fig. 8. TG and DTG curves of ASF, CS, HDPE, and CS-PE-ASF $x$ composites

\section{CONCLUSIONS}

1. The Fourier transform infrared (FT-IR) analysis showed that with the occurrence of red shift, potential hydrogen bonding between cotton stalk (CS) fibers and aluminum silicate fibers (ASF) was formed. The uniform dispersion of inorganic fillers and organic fillers in the polymer matrix combined with the promising interfacial adhesion and interactions played important roles in the improvement of their mechanical strength.

2. When the ASF contents gradually increased, the thermal stabilities also strengthened. In addition, the composites showed enhanced creep and relaxation behavior.

\section{ACKNOWLEDGMENTS}

The authors are grateful for the financial support of the National Key Research and Development Program of China (No. 2018YFD1101001); the distinguished expert of Taishan scholars Shandong Province project and the higher education superior discipline team training program of Shandong Province.

\section{SAFETY STATEMENT}

Asbestos fibers are small and easily dispersed in the air, endangering people's health. Therefore, care should be taken to protect it during operation. 


\section{REFERENCES CITED}

Armistead, C. G., and Hockey, J. A. (1967). "Reactions of chloromethyl silanes with hydrated Aerosil silicas," Transactions of the Faraday Society. 63, 2549. DOI: $10.1039 / \mathrm{tf} 9676302549$

Bucknall, C. B. (2001). "Applications of microscopy to the deformation and fracture of rubber-toughened polymers," Journal of Microscopy (Oxford). 201(2), 221-229. DOI: 10.1046/j.1365-2818.2001.00838.x

Costa, C. S. M. F., Fonseca, A. C., Serra, A. C. and Coelho, J. F. J. (2016). "Dynamic mechanical thermal analysis of polymer composites reinforced with natural fibers," Polym. Rev. 56(2), 362-383. DOI: 10.1080/15583724.2015.1108334

Czigány, T. (2006). "Special manufacturing and characteristics of basalt fiber reinforced hybrid polypropylene composites: Mechanical properties and acoustic emission study," Compos. Sci. Technol. 66(16), 3210-3220. DOI: 10.1016/j.compscitech.2005.07.007

Guo, G., and Kethineni, C. (2020). "Direct injection molding of hybrid polypropylene/ wood-fiber composites reinforced with glass fiber and carbon fiber," The International Journal of Advanced Manufacturing Technology 106(1-2), 201-209. DOI: $10.1007 / \mathrm{s} 00170-019-04572-7$

Hao, X., Zhou, H., Xie, Y., Xiao, Z., Wang, H., and Wang, Q. (2018). "Mechanical reinforcement and creep resistance of coextruded wood flour/polyethylene composites by shell-layer treatment with nano- and micro-SiO 2 particles," Polym. Compos. 40(4), 1576-1584. DOI: 10.1002/pc.24901

Hietala, M., Samuelsson, E., Niinimäki, J., and Oksman, K. (2011). "The effect of presoftened wood chips on wood fibre aspect ratio and mechanical properties of woodpolymer composites," Compos. Part A-Appl. S. 42(12), 2110-2116. DOI: 10.1016/j.compositesa.2011.09.021

Hsiao, M., Liao, S., Yen, M., Liu, P., Pu, N., Wang, C., and Ma, C. M. (2010). "Preparation of covalently functionalized graphene using residual oxygen-containing functional groups," ACS. Appl. Mater. Inter. 2(11), 3092-3099. DOI: $10.1021 / \mathrm{am} 100597 \mathrm{~d}$

Huang, L., Wu, Q., Wang, Q., and Wolcott, M. (2020). "Interfacial crystals morphology modification in cellulose fiber/polypropylene composite by mechanochemical method," Compos. Part A-Appl. S. 130, Article ID 105765. DOI: 10.1016/j.compositesa.2020.105765

Koohestani, B., Ganetri, I., and Yilmaz, E. (2017). "Effects of silane modified minerals on mechanical, microstructural, thermal, and rheological properties of wood plastic composites," Compos. Part B-Eng. 111, 103-111. DOI: 10.1016/j.compositesb.2016.12.021

Li, Q., and Matuana, L. M. (2003). "Surface of cellulosic materials modified with functionalized polyethylene coupling agents," J. Appl. Polym. Sci. 88(2), 278-286. DOI: 10.1002/app.11681

Liu, Y., Guo, L., Wang, W., Sun, Y., and Wang, H. (2019). "Modifying wood veneer with silane coupling agent for decorating wood fiber/high-density polyethylene composite," Constr. Build. Mater. 224, 691-699. DOI: 10.1016/j.conbuildmat.2019.07.090 
Mittal, V., Saini, R. and Sinha, S. (2016). "Natural fiber-mediated epoxy composites - A review," Compos. Part B-Eng. 99, 425-435. DOI: 10.1016/j.compositesb.2016.06.051

Moreno, D. D. P., and Saron, C. (2017). "Low-density polyethylene waste/recycled wood composites," Compos. Struct. 176, 1152-1157. DOI: 10.1016/j.compstruct.2017.05.076

Ou, R., Xie, Y., Wolcott, M. P., Sui, S., and Wang, Q. (2014). "Morphology, mechanical properties, and dimensional stability of wood particle/high density polyethylene composites: Effect of removal of wood cell wall composition," Mater. Design 58, 339-345. DOI: 10.1016/j.matdes.2014.02.018

Oudiani, A. E., Chaabouni, Y., Msahli, S., and Sakli, F. (2011). "Crystal transition from cellulose I to cellulose II in $\mathrm{NaOH}$ treated Agave americana L. fibre," Carbohyd. Polym. 86(3), 1221-1229. DOI: 10.1016/j.carbpol.2011.06.037

Peri, J. B. (1966). "Infrared study of $\mathrm{OH}$ and $\mathrm{NH}_{2}$ groups on the surface of a dry silica aerogel," Journal of Physical Chemistry 70(9), 2937-2945. DOI: 10.1021/j100881a037

Paul, D. K., and Yates, J. T. (1991). "Protection of a rhodium/alumina catalyst under extreme environmental conditions," Journal of Physical Chemistry 95(4), 1699-1703. DOI: $10.1021 / \mathrm{j} 100157 \mathrm{a} 040$

Slavov, S. V., Chuang, K. T., and Sanger, A. R. (1996). "Modification of the surfaces of silica, silica-alumina, and aluminum silicate with chlorotrimethylsilane," Journal of Physical Chemistry 100(40), 16285-16292. DOI: 10.1021/jp961229x

Schwarzkopf, M., Muszyński, L., Hammerquist, C. C., and Nairn, J. A. (2017). "Micromechanics of the internal bond in wood plastic composites: Integrating measurement and modeling," Wood Sci. Technol. 51(5), 997-1014. DOI: 10.1007/s00226-017-0934-5

Sun, H. R., Zhang, S. C., Deng, Z. W., Wang, G. H., Chen, Y. F., and Fang, K. (2014). "Preparation and properties of aluminum silicate fiber mat impregnated silica sol," Key Eng. Mater. 602-603, 126-129. DOI: 10.4028/www.scientific.net/KEM.602603.126

Sun, Y., Guo, L., Liu, Y., Wang, W., and Song, Y. (2019). "Glue wood veneer to woodfiber-high-density-polyethylene composite," Int. J. Adhes. Adhes. 95, Article ID 102444. DOI: 10.1016/j.ijadhadh.2019.102444

Wang, P., Wang, L., Li, D., Huang, Z., Adhikari, B., and Chen, X. D. (2017). "The stressrelaxation behavior of rice as a function of time, moisture and temperature," Int. J. Food Eng. 13(2), 1-10. DOI: 10.1515/ijfe-2016-0162

Wu, Q., Chi, K., Wu, Y., and Lee, S. (2014). "Mechanical, thermal expansion, and flammability properties of co-extruded wood polymer composites with basalt fiber reinforced shells," Mater. Design 60, 334-342. DOI: 10.1016/j.matdes.2014.04.010

Wu, Z., Cui, H., Chen, L., Jiang, D., Weng, L., Ma, Y., Li, X., Zhang, X., Liu, H., Wang, N., et al. (2018). "Interfacially reinforced unsaturated polyester carbon fiber composites with a vinyl ester-carbon nanotubes sizing agent," Compos. Sci. Technol. 164, 195-203. DOI: 10.1016/j.compscitech.2018.05.051

Yang, H. R., Zhao, C. S., and Yu, D. M. (2013). "Research on preparation of new aluminum silicate fiber adiabatic paper," Adv. Mater. Res. 734-737, 2395-2398. DOI: 10.4028/www.scientific.net/AMR.734-737.2395 
Yang, J., Ching, Y., and Chuah, C. (2019). "Applications of lignocellulosic fibers and lignin in bioplastics: A review," Polymers (Basel) 11(5), Article number 751. DOI: 10.3390/polym11050751

Zhang, Q., Cai, H., Ren, X., Kong, L., Liu, J., and Jiang, X. (2017). "The dynamic mechanical analysis of highly filled rice husk biochar/high-density polyethylene composites," Polymers (Basel) 9(11), Article number 628. DOI: 10.3390/polym9110628

Zhang, J., Li, Y., Xing, D., Wang, Q., Wang, H., and Koubaa, A. (2019a). "Reinforcement of continuous fibers for extruded wood-flour/HDPE composites: Effects of fiber type and amount," Constr. Build. Mater. 228, Article ID 116718. DOI: 10.1016/j.conbuildmat.2019.116718

Zhang, Q., Khan, M. U., Lin, X., Cai, H., and Lei, H. (2019b). "Temperature varied biochar as a reinforcing filler for high-density polyethylene composites," Compos. Part B-Eng. 175, Article ID 107151. DOI: 10.1016/j.compositesb.2019.107151

Zhang, Q., Xu, H., Lu, W., Zhang, D., Ren, X., Yu, W., Wu, J., Zhou, L., Han, X., Yi, W. et al. (2020). "Properties evaluation of biochar/high-density polyethylene composites: Emphasizing the porous structure of biochar by activation," Sci. Total Environ. 737, Article ID 139770. DOI: 10.1016/j.scitotenv.2020.139770

Zhao, C. S., Han, W. J., Yu, D. M., and Pang, J. J. (2012). "The design and preparation process of aluminum silicate fiber paperboard machine," Applied Mechanics and Materials 215-216, 235-238. DOI: 10.4028/www.scientific.net/AMM.215-216.235

Article submitted: November 11, 2020; Peer review completed: January 19, 2021;

Revised version received: March 10, 2021; Accepted: March 30, 2021; Published: April $1,2021$.

DOI: $10.15376 /$ biores.16.2.3678-3690 